\title{
BMJ Open Effect of leisure-time physical activity in controlling hypertension: a systematic review and meta-analysis protocol
}

\author{
Md Shariful Islam (D) ,1,2 Md Golam Rabbani (D) , ${ }^{2,3}$ Ammatul Fardousi, ${ }^{3}$ \\ Monaemul Islam Sizear, ${ }^{2}$ KM Saif-Ur-Rahman (i) ${ }^{3,4}$
}

To cite: Shariful Islam M, Rabbani MG, Fardousi A, et al. Effect of leisuretime physical activity in controlling hypertension: a systematic review and metaanalysis protocol. BMJ Open 2021;11:e056270. doi:10.1136/ bmjopen-2021-056270

- Prepublication history and additional supplemental materia for this paper are available online. To view these files, please visit the journal online (http://dx.doi.org/10.1136/ bmjopen-2021-056270)

Received 10 August 2021 Accepted 30 November 2021

D) Check for updates

(c) Author(s) (or their employer(s)) 2021. Re-use permitted under CC BY-NC. No commercial re-use. See rights and permissions. Published by BMJ.

${ }^{1}$ Infectious Diseases Division, icddr,b, Dhaka, Bangladesh ${ }^{2}$ Public Health Foundation, Bangladesh, Dhaka, Bangladesh ${ }^{3}$ Health Systems and Population Studies Division, icddr,b, Dhaka, Bangladesh

${ }^{4}$ Public Health and Health Systems, Nagoya University Graduate School of Medicine, Nagoya, Aichi, Japan

Correspondence to Dr KM Saif-Ur-Rahman; su.rahman@icddrb.org

\section{ABSTRACT}

Introduction Hypertension is a major risk factor for cardiovascular diseases. In 2015, over 1.13 billion individuals worldwide had hypertension. Globally, it results in 10.8 million deaths every year. Around half of the individuals do not continue treatment with medicine to control blood pressure. Physical activity, a nonpharmacological option of treatment, reduces blood pressure. We aim in this research to examine the effect of leisure-time physical activity in controlling blood pressure. Methods and analysis We will follow the Preferred Reporting Items for Systematic Reviews and MetaAnalyses to conduct this study. We will perform a systematic search in Medline/PubMed, Embase, Scopus, Web of Science, Physical Education Index (ProQuest) and CENTRAL (the Cochrane Library). Both experimental and observational studies will be included. The study population would be people with hypertension. Two reviewers will perform screening of the articles, bias assessment and data extraction independently. We will use the Joanna Briggs Institute Critical Appraisal Tools to assess the risk of bias. We will conduct a meta-analysis if it is applicable.

Ethics and dissemination The results will be published in peer-reviewed journals and presented at relevant scientific seminars and conferences. Ethical approval is not applicable.

PROSPERO registration number CRD42021260751.

\section{INTRODUCTION}

Hypertension or elevated blood pressure is a growing global public health concern and is considered a 'silent killer'. 'Around 1.13 billion people were living with hypertension in 2015 globally. ${ }^{2}$ By 2025 , the world will have 1.60 billion people with hypertension. ${ }^{3}$ Hypertension causes clinical and preclinical damage to vital organs, including the brain, heart and kidney. ${ }^{4}$ It resulted in 10.8 million fatalities globally in $2019,{ }^{5}$ with $88 \%$ of death occurring in low-income and middle-income countries (LMICs). ${ }^{6}$ Reducing every $10 \mathrm{~mm}$ $\mathrm{Hg}$ systolic blood pressure and $5 \mathrm{~mm} \mathrm{Hg}$ diastolic pressure decreases cardiovascular event by around a quarter and stroke by around a third. ${ }^{7}$

\section{Strengths and limitations of this study}

- This systematic review will be the first study to synthesise the effects of leisure-time physical activity in controlling blood pressure.

- The study will employ a rigorous search strategy following the gold standard methodology for systematic review, Preferred Reporting Items for Systematic Reviews and Meta-analysis.

- Inclusion and exclusion criteria have been defined to identify all relevant articles regarding the effects of leisure-time physical activity in regulating blood pressure.

- Literature on the English language will only be included in this study which may cause language bias.

Several pharmacological and nonpharmacological treatments are available to manage people with hypertension. Changes in lifestyle, such as regular physical activity and food habits, are considered as the standard and primary treatment for controlling hypertension. ${ }^{89}$ Because non-pharmaceutical interventions such as physical activity, low intake of salt, smoking cessation, lower alcohol drinking and maintaining normal body weight are considered as the cost-effective alternative to control hypertension, ${ }^{10-12}$ whereas postmedication complications and treatment costs may adversely lead to the continuum of medication care of hypertension, particularly in LMICs. ${ }^{13-15}$ American College of Cardiology and American Heart Association also recommended newly diagnosed hypertension with a lower blood pressure threshold can be managed with lifestyle antihypertensive therapy rather than medicines. ${ }^{16}$

Control of hypertension is considered a pathfinder for universal health coverage. ${ }^{17}$ Therefore, one of the global targets for non-communicable diseases is to reduce the prevalence of hypertension by $25 \%$ by 2025 compared with 2010. ${ }^{1}$ Recent empirical epidemiological studies have demonstrated 
the association between leisure-time physical activity and the control of hypertension. ${ }^{18-21}$ Further, various recent experimental evidence from interventional studies have confirmed the association between regular physical exercise and controlling hypertension. ${ }^{22-27}$ Despite the available evidence, there is a dearth of systematic review and meta-analysis as supporting solid evidence on the role for physical activity in controlling high blood pressure integrated and summarised the effects of leisure-time physical activity comprising the epidemiological studies and interventional evidence although such studies are vital for informing policies. Thus, we aim to investigate the effects of leisure-time physical activities in controlling high blood pressure. The study will focus on leisure-time physical activities over other forms of physical activities as knowledge in this domain that is easily translatable to public health messages.

\section{METHODS}

We will conform to the Preferred Reporting Items for Systematic Reviews and Meta-Analyses (PRISMA) to conduct this study. ${ }^{28}$ The study started in July 2021, and the estimated completion date is February 2022.

\section{Study design}

We will include both experimental and observational studies. We will also include all studies conducted at any period.

\section{Study population}

We will include only those studies in which the age of the study population was 18 or above 18 years old. The study population would be patients living with hypertension (controlled or uncontrolled hypertension). We will not limit study participants based on nationality, residency (urban or rural), sex, ethnicity, regions and socioeconomic status. ${ }^{29}$

\section{Study intervention}

Interventions of the selected studies will examine the effect of leisure-time physical activity to control hypertension. We will include studies describing any leisure-time physical activities.

\section{Comparators}

Comparators will be control arms that do not perform any physical activities in leisure time, manage hypertension with any other non-pharmacological or pharmacological interventions.

\section{Outcomes}

The primary outcome will be a reduction of blood pressure. We will assess the outcome of interest from the reported level of blood pressure, systolic or diastolic, or both in selected articles. Secondary outcomes will be obesity indices such as body mass index, waist circumference and waist-hip ratio.

\section{Inclusion criteria}

We will include studies based on the study design, study population, interventions, comparators and outcomes described above.

\section{Exclusion criteria}

We will exclude any reviews, systematic reviews, review of reviews, protocols of trial. We will also exclude finding from books, chapters, comments, perspectives, editorials, letters, correspondences, conference proceedings and opinions. Age of study population less than 18 will be excluded. Moreover, we will exclude unpublished studies and preprints. If any studies are conducted to measure the effect of physical activity to control hypertension, only finding described effect of leisure-time physical activity will be included. If the finding of leisure-time physical activity does not describe separately, the study will be excluded. Studies carried out to examine the effect of leisure-time physical activity to prevent hypertension will also be excluded.

\section{Search strategy}

A comprehensive search will be carried out in electronic bibliographic databases including Medline/PubMed, Embase, Scopus, Web of Science, Physical Education Index (ProQuest) and CENTRAL (the Cochrane Library). We have developed a search strategy for all the above-mentioned databases. We used subject heading terms and keywords related to inclusion and exclusion criteria. Table 1 shows the search strategy for PubMed. Search strategy for the other databases has been added as online supplemental file. Further searches for eligible studies will be conducted by reviewing references within identified articles, relevant review or systematic review articles, relevant journals and different organisational websites. However, the corresponding author will be contacted if required data are not presented, or information is unclear, or full-text articles are not available for any study.

\section{Studies selection}

After retrieving articles, we will import all articles into Rayyan, an open-source software for undertaking screening in systematic reviews. Duplicate articles will be removed. Primarily, two reviewers will screen titles and abstracts independently. The qualified articles will be selected for full-text assessment. At this stage, another set of two reviewers will carry out the full-text review independently for final selection. At any stage, if a disagreement arises, a third reviewer will resolve it through consultation. Figure 1 shows the process of study selection adopted from the PRISMA 2020 statement. ${ }^{28}$

\section{Data extraction}

A data extraction form will be designed and pilot tested before tabulating the data. Two reviewers will extract data independently from the selected studies in a prespecified format. After completing data extraction, both reviewers will critically check data consistency. If any disagreement 
Table 1 Search strategy for PubMed/Medline

Search

number Search terms

\#1 "Leisure time"[tiab] OR "playtime"[tiab] OR "recreation*"[tiab] OR "holiday"[tiab] OR "breaktime"[tiab] OR "free time"[tiab] OR "spare time" [tiab]

\#2 Exercise[Mesh] OR exercise[tiab] OR "physical activ*”[tiab] OR sports[Mesh] OR sports[tiab] OR "Leisure Activities"[Mesh] OR "leisure activ" [tiab] OR walking[Mesh] OR walking[tiab] OR running[Mesh] OR running[tiab] OR jogging[Mesh] OR jogging[tiab] OR baseball[Mesh] OR baseball[tiab] OR basketball[Mesh] OR basketball[tiab] OR bicycling[Mesh] OR bicycling[tiab] OR Boxing [Mesh] OR Boxing [tiab] OR "Cricket Sport"[Mesh] OR cricket[tiab] OR Dancing[Mesh] OR Dancing[tiab] OR football[Mesh] OR football[tiab] OR golf[Mesh] OR golf[tiab] OR Gardening[Mesh] OR Gardening[tiab] OR gymnastics[Mesh] OR gymnastics[tiab] OR mountaineering[Mesh] OR mountaineering[tiab] OR hockey[Mesh] OR hockey[tiab] OR "Martial Arts"[Mesh] OR "Martial Arts"[tiab] OR swimming[Mesh] OR swimming[tiab] OR volleyball[Mesh] OR volleyball[tiab] OR soccer[Mesh] OR soccer[tiab] OR "Water Sports"[Mesh] OR "Water Sports"[tiab] OR wrestling[Mesh] OR wrestling[tiab] OR "weight lifting"[Mesh] OR "weight lifting"[tiab] OR "track and field"[Mesh] OR "track and field"[tiab] OR skiing[Mesh] OR skiing[tiab] OR skating[Mesh] OR skating[tiab] OR "Snow Sports"[Mesh] OR "Snow Sports"[tiab] OR "Racquet Sports"[Mesh] OR "Racquet Sports"[tiab] OR tennis[Mesh] OR tennis[tiab] OR "physical fitness"[Mesh] OR "physical fitness" [tiab] OR "athletic performance"[Mesh] OR "athletic performance" [tiab] OR "Youth Sports"[Mesh] OR "Youth Sports"[tiab] OR "exercise training" [tiab]

\#3 "Hypertension"[Majr] OR "Hypertension/rehabilitation"[Majr] OR "Hypertension/therapy"[Majr] OR Hypertension[tiab]
OR "Blood Pressure" [Mesh] OR "Blood Pressure/classification"[Mesh] OR "Blood Pressure/complications"[Mesh] OR
"Blood Pressure/diagnosis"[Mesh] OR "high blood pressure" [tiab] OR "Blood pressure" [tiab] OR bloodpressure [tiab]
OR "Blood Pressure/metabolism"[Mesh] OR "Blood Pressure/statistics and numerical data"[Mesh] OR "Blood Pressure/
therapy"[Mesh] OR "high blood pressure" [tiab] OR "Blood pressure" [tiab] OR ((arterial OR diastolic OR systolic) AND
pressure) OR "Blood Pressure" [tiab]
\#1 AND \#2 AND \#3

arises, the two reviewers will dissolve through discussion. The extracted data will have the details about the publication, study design, study population, intervention method in intervention and control arms in case of intervention studies, outcomes variables (mean/percentage, SD, ORs, RRs and $95 \%$ CIs). The threshold of leisure-time physical activity will be reported as per the included articles. The corresponding authors will be requested for the missing data.

\section{Assessment of risk of bias}

Two reviewers of the team will independently assess the methodological quality of the selected studies. Reviewers will assess the risk of bias of the selected studies using the Joanna Briggs Institute (JBI) Critical Appraisal Tools as a guide. ${ }^{30} \mathrm{JBI}$ has separate critical appraisal tools for RCTs, quasi-experimental, cohort, cross-sectional and casecontrol studies. We will use specific critical appraisal tools of JBI for specific study designs of selected articles. The studies will then be evaluated as follows: low risk of bias (all items with low risk of bias), high risk of bias (at least one item with the high risk of bias) and uncertain risk of bias (at least one item marked as unclear). A third member will be responsible for addressing any differences that emerged during the risk assessment's interpretation and quality assessment. Furthermore, disagreements between raters will be resolved through discussion.

\section{Data synthesis}

We will perform a meta-analysis to integrate the effect of leisure-time physical activity on R V.4.0 software if data of selected studies permit. We will conduct a meta-analysis if the study design, type of physical activity and reporting outcome variable are similar. A separate meta-analysis will be conducted for RCT and observational studies. We will conduct a dose-response meta-analysis if the finding of dose-response of included studies is available. The outcome measurements (OR, risk ratio, mean difference) will be reported as mentioned in the included articles. We will conduct $\mathrm{I}^{2}$ statistics to determine the heterogeneity of selected studies. We will perform a random effect model meta-analysis to examine the combined effect size if $\mathrm{I}^{2} \leq 50 \% .^{31}$ If we find an $\mathrm{I}^{2}$ value higher than $50 \%$, we will carry out subgroups analysis in this case as main factors may cause heterogeneity such as age, type of study, type of intervention, type of physical activity, settings, status/ severity of hypertension. We will also perform a narrative synthesis of the results. Egger's test will also be used to investigate publication bias. For dichotomous data, the measure of effect size will be reported as point risk ratios, ORs with $95 \%$ CIs. The measure effect size for continuous data will be reported in mean with SD.

\section{Grading of evidence}

The grading of evidence will be graded by two reviewers independently. We will follow the Grading of Recommendations, Assessment, Development and Evaluation (GRADE) approach to assess the grading of evidence. ${ }^{32}$ It will be based on the risk of bias, heterogeneity, indirectness, impression of findings and publications bias. The quality of evidence will be rated as high quality, moderate, low and very low quality, following GRADE guidelines. If there are any disagreements even after discussion among the two reviewers, a third reviewer will resolve through discussion. 


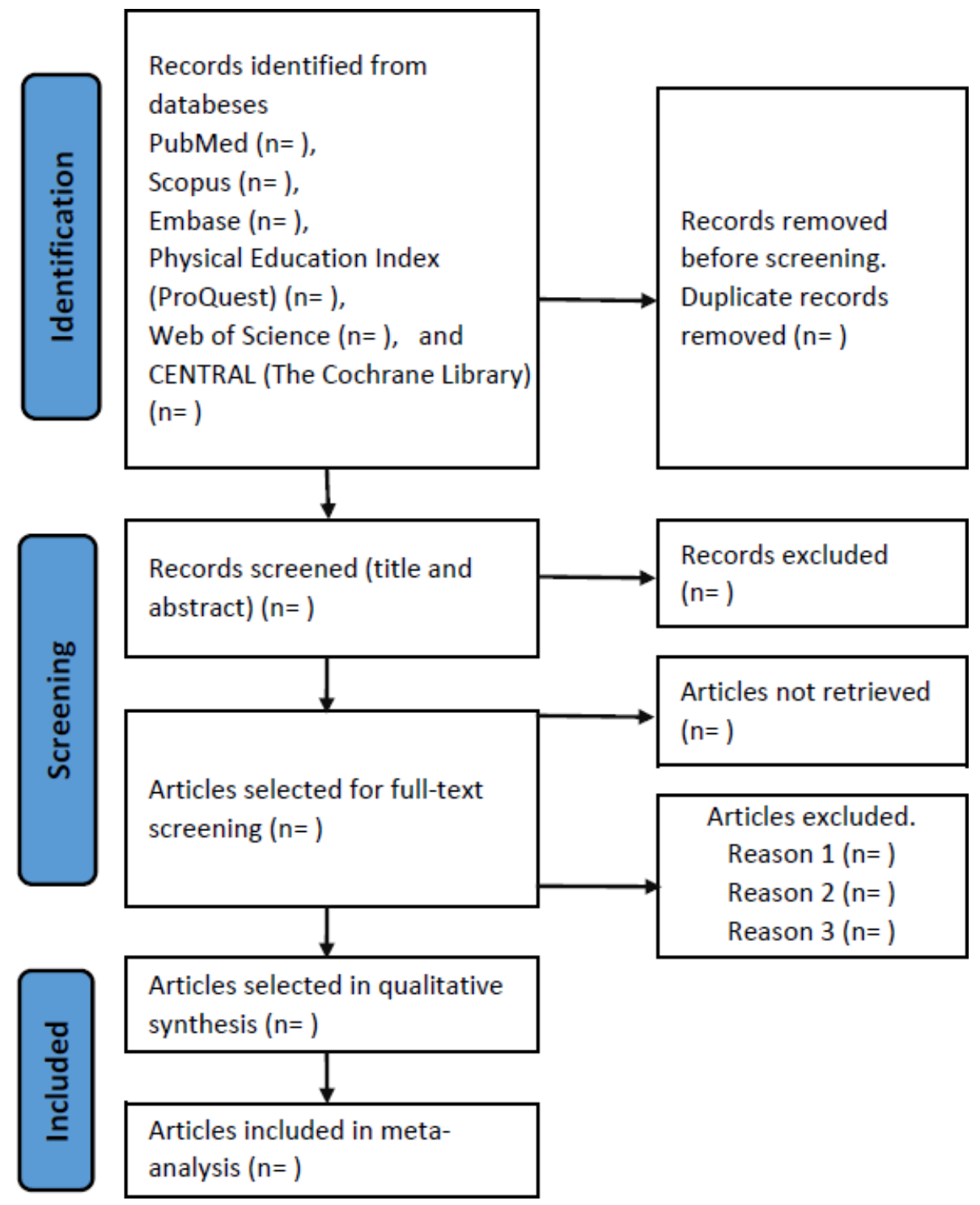

Figure 1 Process of systematic review. ${ }^{28}$

\section{PATIENT AND PUBLIC INVOLVEMENT}

Patients or the public were not involved in the design, or conduct, or reporting, or dissemination plans of our research.

\section{DISCUSSION}

Hypertension is one of the leading causes of poor health status from high-income to low-income countries. ${ }^{33}$ It is evident that controlling high blood pressure decreases the incidence of non-communicable diseases like stroke, heart failure and chronic kidney diseases. ${ }^{74}$ To control hypertension, several pharmacological and nonpharmacological interventions are used. However, the expected outcome is yet to bring in a significant level due to some barriers like treatment adherence, the resistance of drugs, out-of-pocket expenditure. ${ }^{35}$ On the other hand, it is well documented that physical activity improves health outcomes. ${ }^{36-38}$ In the case of controlling hypertension, physical activity has a good potential to reduce the blood pressure level among adults. ${ }^{9}$ A recent study depicted that half of the adults in the united adults have hypertension and leisure-time physical activity can improve the health condition though little is known about the level of the impact of this. ${ }^{39}$ However, no specific systematic review is found that concentrates on the effect of leisure-time physical activity to control hypertension.

Considering the importance of the issue and the limitation of the significant evidence, this study investigates how leisure-time physical activity can be an effective measure to control growing hypertension. The study poses some potential significance and implications that contribute to taking the evidence-based policy formulation by the decision-makers to deal with hypertension. The study will help researchers to use the findings and recommendations for further research. To disseminate the findings of this research among policymakers, physicians and public health researchers, we will use a wider arena such as journals, seminars, social media and daily newspapers. 
This study does not limit to geography, residency and study period to include relevant studies in this review. A rigorous search strategy will be followed where metaanalysis will be carried out. These criteria help to improve the study synthesis. However, published articles based on the English language would be included, which is the only limitation of this protocol. This study does not include other language articles.

\section{ETHICS AND DISSEMINATION}

The findings will be disseminated in peer-reviewed journals and presented at scientific seminars and conferences relevant to the field. Individual patient information is not used. We do not need ethical approval to conduct this study.

Acknowledgements The authors would like to acknowledge the contribution of the current donors providing unrestricted support to icddr,b that include: the Governments of Bangladesh, Canada, Sweden and the UK. We gratefully acknowledge these donors for their support and commitment to icddr,b's research efforts.

Contributors KMS-U-R and MSI conceptualised the study, MGR and KMS-U-R contributed to the methodology section, MGR and MSI developed a search strategy. MSI, AF, MIS and MGR drafted the protocol. KMS-U-R critically reviewed and finalised the manuscript. KMS-U-R is the guarantor of the systematic review. All authors approved the publication of the protocol.

Funding The authors have not declared a specific grant for this research from any funding agency in the public, commercial or not-for-profit sectors.

Competing interests None declared.

Patient consent for publication Not applicable.

Provenance and peer review Not commissioned; externally peer reviewed.

Supplemental material This content has been supplied by the author(s). It has not been vetted by BMJ Publishing Group Limited (BMJ) and may not have been peer-reviewed. Any opinions or recommendations discussed are solely those of the author(s) and are not endorsed by BMJ. BMJ disclaims all liability and responsibility arising from any reliance placed on the content. Where the content includes any translated material, BMJ does not warrant the accuracy and reliability of the translations (including but not limited to local regulations, clinical guidelines, terminology, drug names and drug dosages), and is not responsible for any error and/or omissions arising from translation and adaptation or otherwise.

Open access This is an open access article distributed in accordance with the Creative Commons Attribution Non Commercial (CC BY-NC 4.0) license, which permits others to distribute, remix, adapt, build upon this work non-commercially, and license their derivative works on different terms, provided the original work is properly cited, appropriate credit is given, any changes made indicated, and the use is non-commercial. See: http://creativecommons.org/licenses/by-nc/4.0/.

\section{ORCID iDs}

Md Shariful Islam http://orcid.org/0000-0002-3015-0830

Md Golam Rabbani http://orcid.org/0000-0003-3166-0397

KM Saif-Ur-Rahman http://orcid.org/0000-0001-8702-7094

\section{REFERENCES}

1 World Health Organization. Fact sheets. hypertension, 2021. Available: https://www.who.int/news-room/fact-sheets/detail/ hypertension

2 NCD Risk Factor Collaboration (NCD-RisC). Worldwide trends in blood pressure from 1975 to 2015: a pooled analysis of 1479 population-based measurement studies with $19 \cdot 1$ million participants. Lancet 2017;389:37-55.

3 Kearney PM, Whelton M, Reynolds K, et al. Global burden of hypertension: analysis of worldwide data. Lancet 2005;365:217-23.

4 Mensah GA. Hypertension and Target Organ Damage: Don't Believe Everything You Think! Ethn Dis 2016;26:275-8.
5 GBD 2019 Risk Factors Collaborators. Global burden of 87 risk factors in 204 countries and territories, 1990-2019: a systematic analysis for the global burden of disease study 2019. Lancet 2020;396:1223-49.

6 Zhou B, Perel P, Mensah GA, et al. Global epidemiology, health burden and effective interventions for elevated blood pressure and hypertension. Nat Rev Cardiol 2021;18:785-802.

7 Law MR, Morris JK, Wald NJ. Use of blood pressure lowering drugs in the prevention of cardiovascular disease: meta-analysis of 147 randomised trials in the context of expectations from prospective epidemiological studies. BMJ 2009;338:b1665.

8 Yang MH, Kang SY, Lee JA, et al. The effect of lifestyle changes on blood pressure control among hypertensive patients. Korean J Fam Med 2017;38:173-80.

9 Pescatello LS, Buchner DM, Jakicic JM, et al. Physical activity to prevent and treat hypertension: a systematic review. Med Sci Sport Exerc 2019;51:1314-23.

10 Akonobi A, Khan J. A systematic review of Randomized Controlled Trials about some non- pharmacological interventions for treatment of hypertension : Physical exercise, sodium restriction, weight and alcohol use reduction. $J$ Heal Soc Sci 2019:17-42.

11 Fu J, Liu Y, Zhang L, et al. Nonpharmacologic interventions for reducing blood pressure in adults with prehypertension to established hypertension. J Am Heart Assoc 2020;9:e016804.

12 Gupta R, Guptha S. Strategies for initial management of hypertension. Indian J Med Res 2010;132:531-42.

13 Oyando R, Njoroge M, Nguhiu P, et al. Patient costs of hypertension care in public health care facilities in Kenya. Int $\mathrm{J}$ Health Plann Manage 2019;34:e1166-78.

14 MacMahon S, Alderman MH, Lindholm LH, et al. Blood-pressurerelated disease is a global health priority. Am $J$ Hypertens 2008;21:843-4.

15 Murphy A, Jakab M, McKee M, et al. Persistent low adherence to hypertension treatment in Kyrgyzstan: how can we understand the role of drug affordability? Health Policy Plan 2016;31:1384-90.

16 Whelton PK, Carey RM, Aronow WS. Acc/aha/aapa/abc/acpm/ ags/APhA/ASH/ASPC/nma/pcna guideline for the prevention, detection, evaluation, and management of high blood pressure in adults: a report of the American College of Cardiology/ American heart association. Task force on clinical practi. KIDNEYS 2018;7:68-74.

17 Frieden TR, Varghese CV, Kishore SP, et al. Scaling up effective treatment of hypertension $-\mathrm{A}$ pathfinder for universal health coverage. J Clin Hypertens 2019;21:1442-9.

18 Diaz KM, Shimbo D. Physical activity and the prevention of hypertension. Curr Hypertens Rep 2013;15:659-68.

19 Haapanen N, Miilunpalo S, Vuori I, et al. Association of leisure time physical activity with the risk of coronary heart disease, hypertension and diabetes in middle-aged men and women. Int J Epidemiol 1997;26:739-47.

20 Pitanga FJG, Matos SMA, Almeida MdaCC, et al. Association between leisure-time physical activity and sedentary behavior with cardiometabolic health in the ELSA-Brasil participants. SAGE Open Med 2019;7:205031211982708.

21 Yoon J-H, So W-Y. Association between leisure-time physical activity and hypertension status in Korean adults. Salud Publica Mex 2013;55:492-7.

22 Arija V, Villalobos F, Pedret R, et al. Physical activity, cardiovascular health, quality of life and blood pressure control in hypertensive subjects: randomized clinical trial. Health Qual Life Outcomes 2018;16:184.

23 Medina C, Janssen I, Barquera S, et al. Occupational and leisure time physical inactivity and the risk of type II diabetes and hypertension among Mexican adults: a prospective cohort study. Sci Rep 2018;8:5399.

24 Byambasukh O, Snieder H, Corpeleijn E. Relation Between Leisure Time, Commuting, and Occupational Physical Activity With Blood Pressure in 125402 Adults: The Lifelines Cohort. J Am Heart Assoc 2020;9:e014313.

25 Lamina S, Okoye CG, Hanif SM. Randomised controlled trial: effects of aerobic exercise training programme on indices of adiposity and metabolic markers in hypertension. J Pak Med Assoc 2013;63:680-7.

26 Herrod PJJ, Lund JN, Phillips BE. Time-efficient physical activity interventions to reduce blood pressure in older adults: a randomised controlled trial. Age Ageing 2021;50:980-4.

27 Mattila R, Malmivaara A, Kastarinen M, et al. Effectiveness of multidisciplinary lifestyle intervention for hypertension: a randomised controlled trial. J Hum Hypertens 2003;17:199-205.

28 Page MJ, McKenzie JE, Bossuyt PM, et al. The PRISMA 2020 statement: an updated guideline for reporting systematic reviews. BMJ 2021;372:n71. 
29 Liu X, Zhang D, Liu Y, et al. Dose-Response association between physical activity and incident hypertension: a systematic review and meta-analysis of cohort studies. Hypertension 2017;69:813-20.

30 Porritt K, Gomersall J, Lockwood C. JBI's systematic reviews: study selection and critical appraisal. Am J Nurs 2014;114:47-52.

31 Rodriguez AJ, Mousa A, Ebeling PR, et al. Effects of vitamin $D$ supplementation on inflammatory markers in heart failure: a systematic review and meta-analysis of randomized controlled trials. Sci Rep 2018;8:1-8.

32 Guyatt GH, Oxman AD, Vist GE, et al. Grade: an emerging consensus on rating quality of evidence and strength of recommendations. $B M J$ 2008;336:924-6.

33 Chow CK, Teo KK, Rangarajan S, et al. Prevalence, awareness, treatment, and control of hypertension in rural and urban communities in high-, middle-, and low-income countries. JAMA 2013;310:959-68.

34 GBD 2017 Risk Factor Collaborators. Global, regional, and national comparative risk assessment of 84 behavioural, environmental and occupational, and metabolic risks or clusters of risks for 195 countries and territories, 1990-2017: a systematic analysis for the global burden of disease study 2017. Lancet 2018;392:1923-94.

35 Khatib R, Schwalm J-D, Yusuf S, et al. Patient and healthcare provider barriers to hypertension awareness, treatment and follow up: a systematic review and meta-analysis of qualitative and quantitative studies. PLoS One 2014;9:e84238.

36 ISPAH International Society for Physical Activity and Health. The Bangkok Declaration on physical activity for global health and sustainable development. Br J Sports Med 2017:51:1389-91.

37 Sallis JF, Bull F, Guthold R, et al. Progress in physical activity over the Olympic quadrennium. Lancet 2016;388:1325-36.

38 World Health Organization. Global recommendations on physical activity for health. World Health Organization, 2010.

39 Al Kibria GM, Das GR, Crispen R. Association of leisure-time physical activity with perceived general health status among hypertensive people: an analysis of NHANES 2015-18. J Hum Hypertens 2021:1-9. 